\title{
MARINE BIOLOGY
}

\section{Twilight budget in balance}

Large quantities of carbon are captured from the atmosphere and converted into organic matter in the sunlit waters of the surface ocean. A significant fraction of this material sinks down from the surface waters into the twilight zone, that dimly lit stretch of the ocean that extends from the base of the photosynthetic zone down to 1,000 metres depth. Resolving the fate of the material that enters this zone is a key challenge in carbon cycle research.

The organic matter that enters the twilight zone serves as a source of food for the zooplankton - multicellular marine organisms that populate much of the world ocean - and microbes that reside in these waters. However, the amount of carbon consumed by these organisms exceeds that supplied by sinking particles, according to a number of observational studies. This apparent mismatch between supply and demand has proved tricky to resolve.

Sarah Giering and colleagues balance the carbon budget in the twilight zone at the Porcupine Abyssal Plain site in the northeastern Atlantic Ocean (Nature http://dx.doi.org/10.1038/nature13123; 2014). They constructed a comprehensive

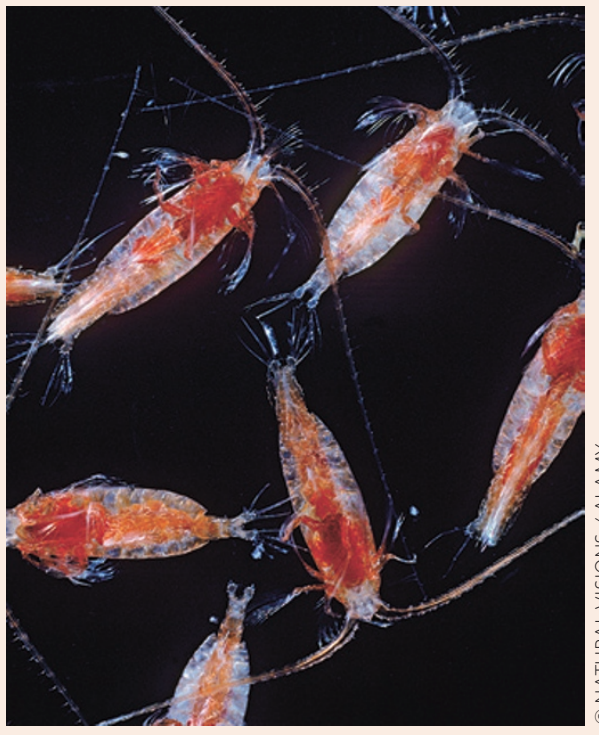

supply and demand in these waters can be attributed to improved estimates of carbon inputs, and the consideration of community respiration as opposed to carbon demand.

Giering etal. find that the majority of the carbon that enters the twilight zone floats down in the form of large, fast-sinking particles, ideal fodder for zooplankton. However, using a simple model, they show that more than $30 \%$ of the organic matter fragmented and consumed by these creatures is ejected into the surrounding water column in the form of slow-sinking particles, ripe for colonization by microbes. Indeed, microbial respiration accounts for most of the carbon respired and ultimately lost from these waters.

Marine biota mediate the fate of the carbon that enters the twilight waters of the world ocean, with consequences for ocean carbon storage and release. Although much remains to be learnt about carbon dynamics in these waters, it seems clear that ecological interactions play an important role in shaping twilight zone carbon consumption and release. carbon budget for the these waters - using oceanographic measurements, satellite data and a food web model - and show that the net input of organic carbon into the twilight zone matches that lost through the respiration of organic matter, within the uncertainty range of the observations. They suggest that the reconciliation of carbon

\section{Desert dust and monsoon rain}

The climate regimes of monsoon regions and deserts are connected. Satellite data and numerical experiments reveal that an increase in dust aerosol loading over the Arabian Sea and West Asia can lead to enhanced summer monsoon rainfall over central India on timescales of days to weeks.

\section{William Lau}

F or centuries, inhabitants of the Indian subcontinent have known that heavy dust events brought on by strong winds occur frequently in the pre-monsoon season, before the onset of heavy rain. Yet scientists have never seriously considered the possibility that natural dust can affect monsoon rainfall. Up to now, most studies of the impacts of aerosols on Indian monsoon rainfall have focused on anthropogenic aerosols in the context of climate change. However, a few recent studies have shown that aerosols from anthropogenic and natural sources over the Indian subcontinent may affect the transition from break to active monsoon phase ${ }^{1,2}$ on short timescales of days to weeks. As they report in Nature Geoscience, Vinoj and colleagues ${ }^{3}$ now show that desert dust aerosols over the Arabian Sea and West Asia can strengthen the summer monsoon over the Indian subcontinent in a matter of days.

Prevailing low-level winds carry dust particles from the deserts of West Asia, the Middle East and North Africa across the Arabian Sea to the Indian subcontinent each year, before the onset of the summer monsoon $^{4}$ (Fig. 1). As the monsoon develops, these low-level winds strengthen and transport more dust from the desert regions. As a result, dust levels over the Arabian Sea, northwestern India, the IndoGangetic Plain and the Himalaya foothills peak during the late boreal spring and early summer months. As the monsoon season progresses, atmospheric dust loading diminishes over India because of wash-out by heavy rainfall and weakening winds.

Airborne dust particles can cool the underlying surface by reducing the total amount of sunlight reaching the surface through scattering and absorbing incoming 